\title{
Association of STAT4 gene single nucleotide polymorphisms with Iranian juvenile-onset systemic lupus erythematosus patients
}

\author{
Arash Salmaninejad ${ }^{1,2}$, Mahdi Mahmoudi ${ }^{1,3}$, Saeed Aslani ${ }^{1,3}$, Shiva Poursani ${ }^{1}$, Vahid \\ Ziaee $^{4,5}$, Nima Rezaei $6,7,8$ \\ ${ }^{1}$ Rheumatology Research Center, Tehran University of Medical Sciences, Tehran, Iran ; ${ }^{2}$ Student Research Committee, \\ Medical Genetics Research Center, Mashhad University of Medical Sciences, Mashhad, Iran; ${ }^{3}$ Rheumatology Expert Group \\ (REG), Universal Scientific Education and Research Network (USERN), Tehran, Iran; ${ }^{4}$ Pediatric Rheumatology Research \\ Group, Rheumatology Research Center; ${ }^{5}$ Division of Pediatric Rheumatology, Pediatrics Center of Excellence; ${ }^{6}$ Department \\ of Immunology, School of Medicine; ${ }^{7}$ Research Center for Immunodeficiencies, Children's Medical Center, Tehran University \\ of Medical Sciences, Tehran, Iran; ${ }^{8}$ Network of Immunity in Infection, Malignancy and Autoimmunity (NIIMA), Universal \\ Scientific Education and Research Network (USERN), Sheffield, UK. \\ E-mail: rezaeinima@tums.ac.ir and mahmoudim@tums.ac.ir \\ Received 9th January 2017, Revised 12th March 2017, Accepted: 12th March 2017
}

SUMMARY: Salmaninejad A, Mahmoudi M, Aslani S, Poursani S, Ziaee V, Rezaei N. Association of STAT4 gene single nucleotide polymorphisms with Iranian juvenile-onset systemic lupus erythematosus patients. Turk J Pediatr 2017; 59: 144-149.

Juvenile-onset systemic lupus erythematosus (JSLE) is a complex autoimmune disease, characterized by multi-organ involvement. Single nucleotide polymorphisms (SNPs) of signal transducer and activator of transcription 4 (STAT4) gene have been reported to have relationship with the risk of several autoimmune diseases. Studies have provided evidence that STAT4 may participate in the pathogenesis of JSLE. Therefore, we aimed to evaluate the association of STAT4 SNPs with JSLE in Iranian population. In this case-control study, two SNPs of STAT4 gene, including rs7574865 and rs7601754 were genotyped in 50 Iranian JSLE patients and 281 matched healthy individuals using real-time PCR allelic discrimination approach. Our experiments demonstrated that $G$ and $T$ alleles of rs7574865 SNP had similar distribution between patients and controls $(P=0.16)$. Additionally, differences in frequency of GG, GT, and TT genotypes $(P=0.14,0.29$, and 0.54 , respectively) were not significant. Likewise, A and G alleles, as well as genotypes of rs7601754 SNP did not show significant differences between JSLE patients and healthy individuals. Lack of association of rs7574865 and rs7601754 SNPs in STAT4 gene with susceptibility to JSLE in Iranian population, despite their association with the risk of adult SLE in the same population, implicates on difference of genetic background of JSLE and SLE.

Key words: children, juvenile systemic lupus erythematosus, SLE, SNP, STAT4.

Systemic lupus erythematosus (SLE) is an overwhelming chronic, inflammatory, and complex systemic autoimmune disorder that characterized by multi-organ involvement. In spite of unknown etiopathology, it has been postulated that a combination of genetic background, environmental and hormonal factors may contribute to the initiation and development of SLE. SLE is primarily manifested by autoantibodies against nuclear antigens and immune complex deposition throughout the tissues, which subsequently cause the autoimmune reactions in the disease ${ }^{1}$. SLE affects primarily females during adulthood. Nonetheless, approximately less than $20 \%$ of SLE cases of all types initiates in teenagers before the age of 16 years, defined as JSLE, which has less discrepancies in the disease gender basis of female predominance as compared to adults ${ }^{2,3}$.

Signal transducer and activator of transcription 
factor 4 (STAT4) is involved in inflammatory responses through immune system activation. STAT4 also plays a role in differentiation of helper $1 \mathrm{~T}$ cells from naive $\mathrm{CD}^{+}{ }^{+} \mathrm{T}$ cells ${ }^{4}$. Gene variations in STAT4 have been associated with several autoimmune disorders. The rs7574865 G/T SNP occurs in intron 3 of STAT4 gene, and has been associated with juvenile idiopathic arthritis (JIA) in non-Hispanic white population ${ }^{5}$. Genome-wide association study (GWAS) and a meta-analysis have reported that STAT4 rs7574865 T allele confers susceptibility to JIA, SLE, rheumatoid arthritis (RA), type 1 diabetes (T1D), systemic sclerosis (SSc), primary antiphospholipid syndrome (APS), inflammatory bowel diseases-ulcerative colitis (IBD-UC), and primary Sjogren's syndrome (pSS) 6, 7 . Moreover, the minor C allele of STAT4 rs7601754 SNP was shown to be a protective allele in SLE, whereas the major $\mathrm{T}$ allele might be a risk allele for SLE susceptibility ${ }^{8}$. In addition, the exploration of STAT4 rs7574865 and rs7601754 SNPs in Iranian SLE patients revealed a significant association between disease and rs7574865 SNP9 9

Previous studies verify the hypothesis of association between STAT4 gene polymorphisms and a variety of autoimmune diseases ${ }^{10,11}$. Considering the autoimmune nature of JSLE, it appears that the functional STAT4 rs7574865 (and rs7601754) SNP, which is commonly related to adult-onset SLE, may also be associated with JSLE. This is the first study that evaluated probable associations of STAT4 gene rs7574865 and rs7601754 SNPs with JSLE predisposition in Iranian pediatric patients.

\section{Participants and Methods}

\section{Patient and control subjects}

The patients were brought together from Rheumatology Clinic of the Children's Medical Center Hospital, the Pediatrics Center of Excellence in Iran and diagnosis of JSLE was conducted according to revised criteria of the American College of Rheumatology (ACR) 12. For genotyping, 50 JSLE patients (Table I) and 281 healthy age and sex matched volunteers were involved in the study. None of the healthy controls had autoimmune disease neither in themselves nor their immediate family members. The Human Research Ethics Committees from the Tehran University of
Medical Sciences authorized this study. Written informed consent was taken by all participants. Blood samples from patients were obtained during clinical diagnosis; as such samples were obtained from healthy controls. About $5 \mathrm{ml}$ of blood from each subject was collected in EDTA-anticoagulated tubes using venipuncture. DNA from the samples was extracted using phenol-chloroform method.

\section{Positional candidate SNP selection and real-time PCR genotyping}

All the samples were genotyped for rs7574865 and rs7601754, using real-time allelic discrimination Taq-Man assays (Applied Biosystems, Foster City, USA). Briefly, all PCR reaction mixtures contained approximately 25-75 ng of DNA, $10 \mu l$ Taq-Man Master Mix containing Taq DNA polymerase and dNTPs (Applied Biosystems, Foster City, USA), 0.5 $\mu \mathrm{l}$ Taq-Man Genotyping Assay mix containing primers and FAM or VIC labeled probes (Applied Biosystems, Foster City, USA), and distilled water for a final volume of $20 \mu \mathrm{l}$. Real-time allelic discrimination PCR was performed by StepOnePlus Real-Time PCR system (Applied Biosystems, Foster City, USA). The real-time PCR conditions were: initially $60^{\circ} \mathrm{C}$ for 30 seconds and then $95^{\circ} \mathrm{C}$ for 10 mins, and subsequently 40 cycles of amplification $\left(95^{\circ} \mathrm{C}\right.$ for 15 seconds and $60^{\circ} \mathrm{C}$ for $1 \mathrm{~min}$ ), and finally $60^{\circ} \mathrm{C}$ for 30 seconds.

\section{Statistical analysis}

Genotype and allelic distribution between case and control groups were implemented by ChiSquare test. Pearson's $\chi 2$-tests were applied to test for significance differences of both genotype and allele frequencies between two groups. All probability values were calculated from two-tailed test. Moreover, the odds ratio (OR) and 95\% confidence interval (CI) were calculated. The genotype distributions of SNPs were tested for deviation from Hardy-Weinberg equilibrium in controls. Statistical analysis was performed using the SPSS for Windows (version 22.0, IBM SPSS Inc., USA). Additionally, the SHEsis online software was applied for analyzing the haplotype and genotype, and also Hardy-Weinberg equilibrium ${ }^{13}$. A p value $<0.05$ was considered significant. 


\section{Results}

\section{Alleles and Genotypes Frequencies}

The distribution of the genotypes for both rs7574865 and rs7601754 in control group did not reveal any significant deviation from the Hardy-Weinberg equilibrium (Table II).

In respect of rs7574865 SNP (Table II), G allele was found in $61 \%$ of patients and $68.1 \%$ of controls; due to equal frequency between two groups, no significant difference was observed $(\mathrm{OR}=0.73,95 \% \mathrm{CI}: 0.47-1.13$; $\mathrm{P}=0.16)$. Moreover, there was no significant $(\mathrm{OR}=1.36,95 \% \mathrm{CI}: 0.88-2.12 ; \mathrm{P}=0.16)$ difference of $\mathrm{T}$ allele distribution between patients (39\%) and healthy controls (31.9\%). The GG genotype was seen in $38 \%$ of JSLE patients and $49.1 \%$ of the healthy subjects, and had no significant difference between patients and controls $(\mathrm{OR}=0.63,95 \% \mathrm{CI}$ : 0.34-1.17; $\mathrm{P}=0.14$ ). Meanwhile both GT and TT genotypes had no significant difference between the two groups $(\mathrm{OR}=1.38,95 \% \mathrm{CI}$ : $0.75-2.53 ; \mathrm{P}=0.29$ and $\mathrm{OR}=1.29,95 \% \mathrm{CI}: 0.56-2.98 ; \mathrm{P}=0.54)$.
The A allele for rs7601754 polymorphism in STAT4 gene (Table II) was found in $84 \%$ of patients and closely in $80.1 \%$ of healthy controls, resulting in insignificant difference between these two groups $(\mathrm{OR}=1.30,95 \%$ CI: 0.73-2.31; $P=0.36)$. Although being a less common allele, the $G$ allele was found with nearly same frequency in $16 \%$ of JSLE patients and $19.9 \%$ of controls, hence the distribution difference was not significant $(\mathrm{OR}=0.76,95 \%$ CI: 0.43-1.36; $P=0.36$ ). The AA genotype for above mentioned SNP was highly frequent, but similarly observed in both patients and control groups ( $72 \%$ vs. $66.2 \%$, respectively) resulting in no significant difference $(\mathrm{OR}=1.31$, 95\% CI: $0.67-2.55 ; P=0.42)$. The GG genotype was rarely seen in patients and controls (4\% vs. $5.9 \%$, respectively) with no significant difference $(\mathrm{OR}=0.66,95 \% \mathrm{CI}$ : 0.14-2.99; $P=0.59)$. Additionally, The AG genotype represented no significant difference between two study subjects $(\mathrm{OR}=0.81,95 \% \mathrm{CI}$ : $0.40-$ 1.64; $P=0.56)$.

Table I. Demographic Characteristics and Laboratory Measurements of JSLE Patients.

\begin{tabular}{ll}
\hline Specification (50 JSLE Patients) & Results \\
\hline Age (years) & $11.06 \pm 3.45$ \\
Male, n (\%) & $7(14)$ \\
Female, n (\%) & $43(86)$ \\
Disease duration (years) & $4.08 \pm 2.42$ \\
Arthritis, n (\%) & $12(24)$ \\
Malar rash, n (\%) & $33(66)$ \\
Oral ulcer, n (\%) & $25(50)$ \\
Discoid rash, n (\%) & $2(4)$ \\
Photosensitivity, n (\%) & $37(74)$ \\
Pleuritis, n (\%) & $3(6)$ \\
Precarditis, n (\%) & $2(4)$ \\
Hematological involvement, n (\%) & $17(34)$ \\
Renal involvement, n (\%) & $23(46)$ \\
CNS involvement, n (\%) & $6(12)$ \\
ANA positivity, n (\%) & $45(90)$ \\
Anti-dsDNA positivity, n (\%) & $39(78)$ \\
Anti-Sm* positivity, n (\%) & $1 / 9(11.1)$ \\
\hline
\end{tabular}

*This group consists of nine SLE patients.

ANA: antinuclear antibody; anti-dsDNA: anti-double stranded DNA; anti Sm: anti-Smith antibody; CNS: central nervous system. 
Table II. Allele and Genotype Distribution of STAT4 Gene SNPs in JSLE Patients and Healthy Subjects.

\begin{tabular}{llllll}
\hline dbSNP & $\begin{array}{l}\text { Alleles/ } \\
\text { Genotypes }\end{array}$ & $\begin{array}{l}\text { Case }(\mathrm{n}=50) \\
\mathrm{N}(\%)\end{array}$ & $\begin{array}{l}\text { Control }(\mathrm{n}=281) \\
\mathrm{N}(\%)\end{array}$ & $\mathrm{P}$ & OR (95\% CI) \\
\hline rs7574865 & G & $61(61)$ & $383(68.1)$ & 0.16 & $0.73(0.47-1.13)$ \\
& T & $39(39)$ & $179(31.9)$ & 0.16 & $1.36(0.88-2.12)$ \\
& GG & $19(38)$ & $138(49.1)$ & 0.14 & $0.63(0.34-1.17)$ \\
& GT & $23(46)$ & $107(38.1)$ & 0.29 & $1.38(0.75-2.53)$ \\
& TT & $8(16)$ & $36(12.8)$ & 0.54 & $1.29(0.56-2.98)$ \\
HWE & & & 0.06 & & \\
rs7601754 & A & $84(84)$ & $436(80.1)$ & & \\
& G & $16(16)$ & $108(19.9)$ & 0.36 & $1.30(0.73-2.31)$ \\
& AA & $36(72)$ & $180(66.2)$ & 0.36 & $0.76(0.43-1.36)$ \\
& AG & $12(24)$ & $76(27.9)$ & 0.56 & $1.31(0.67-2.55)$ \\
& GG & $2(4)$ & $16(5.9)$ & $0.81(0.40-1.64)$ \\
HWE & & & 0.07 & & $0.66(0.14-2.99)$ \\
\hline
\end{tabular}

CI: confidence interval; HWE: Hardy-Weinberg equilibrium; OR: Odds ratio

\section{Haplotype Frequencies}

Table III shows haplotype frequencies in patients with JSLE and controls. Regarding the haplotype analysis (rs7574865 G/T - rs7601754 A/G), no significant distribution of the GA, GG, TA, TG haplotypes was observed between JSLE patients and healthy controls.

\section{Discussion}

STAT4 gene encodes a transcription factor, which contributes differentiation of $\mathrm{T}$ helper 1 cells and promotes the activity of genes in these cells to produce specific cytokines ${ }^{4}$. Functioning as transcription factor, STAT4 participates in responses to interleukin (IL)12 in lymphocytes and facilitates mRNA transcription of IL-23, interferon (IFN) $-\gamma$, and IL-17 genes in immune cells in normal and abnormal immune responses ${ }^{14,15}$.

SLE is a multifactorial autoimmune disorder with complex genetic inheritance. Numerous liable genes have been recognized that have a role as an augmentative element in disease risk $^{16}$. In the genetics point of view, our previous studies demonstrated the role of gene polymorphisms in susceptibility to JSLE. A positive association between the promoter sequence of the IL- $1 \beta$ gene at position -511 for TT genotype and JSLE risk was recognized. On the other hand, a significant negative association was found between the TC genotype at the mentioned position in the IL-1 $\beta$ gene and JSLE patients compared with healthy subjects. However, none of the polymorphisms in $I L 1 \alpha$, $I L-6, I L 1 R$, and ILIRa genes were significantly associated with JSLE susceptibility ${ }^{17}$. Moreover, Pontillo et al. ${ }^{18}$ demonstrated IL-1 $\beta$ involvement in the pathogenesis of SLE, among the other analyzed polymorphisms in inflammasome genes (NLRP1, NLRP3, CARD8, TNFAIP3). Gene polymorphisms of two anti-inflammatory cytokines, including IL-10 and transforming growth factor beta (TGF- $\beta$ ) were investigated, resulting in significant IL-10, but not TGF- $\beta$, gene SNP association with JSLE ${ }^{19}$. Association of polymorphisms in programmed cell death $1(P D C D 1)^{20,21}$, protein tyrosine phosphatase non-receptor type $22(P T P N 22)^{22,23}$, and IL-424 genes, as well as C1q rs292001 SNP25 has been observed in JSLE patients. In our previous study, we evaluated the association of programmed cell death1( also called PD-1) gene polymorphisms with JSLE susceptibility in the Iranian population and our finding supported the influence of the PD1.1 SNP on the development of juvenile systemic lupus erythematosus (JSLE) in the Iranian population ${ }^{21,26}$.

Accumulating investigations have disclosed association of STAT4 polymorphisms with autoimmune disorders. STAT4 rs7574865 SNP has been identified repetitively to be 
Table III. Overall Haplotype Distribution of the STAT4 SNPs According to Haploview in JSLE and Healthy Groups.

\begin{tabular}{|c|c|c|c|c|c|c|}
\hline \multicolumn{3}{|c|}{ Block 1 Haplotypes } & \multicolumn{2}{|c|}{ Haplotype frequencies } & \multirow[b]{2}{*}{$\mathrm{OR}^{*}\left(95 \% \mathrm{CI}^{*}\right)$} & \multirow[b]{2}{*}{$\mathrm{p}$} \\
\hline ב) & $\begin{array}{l}n \\
6 \\
0 \\
+ \\
1 \\
10 \\
0 \\
0\end{array}$ & 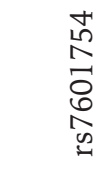 & Cases & Controls & & \\
\hline 1 & G & A & $45.00(45)$ & $262.47(40.6)$ & $0.87(0.56-1.34)$ & 0.53 \\
\hline 2 & G & G & $16.00(16)$ & $106.53(19.6)$ & $0.78(0.43-1.38)$ & 0.39 \\
\hline 3 & $\mathrm{~T}$ & A & $39.00(39)$ & $173.53(31.9)$ & $1.36(0.87-2.11)$ & 0.17 \\
\hline 4 & $\mathrm{~T}$ & G & $0.00(0)$ & $1.47(0.10)$ & $\mathrm{NC}$ & $\mathrm{NC}$ \\
\hline
\end{tabular}

*OR: odds ratio; CI: confidence interval; NC: not calculated

a susceptibility variation for autoimmune diseases like SLE7,8,27. As well as, STAT4 rs7574865 polymorphism has been recognized as a contributing variant in adult SLE of Iranian population ${ }^{9}$. However, no significant association was seen between rs7574865 in STAT4 gene and predisposition toward Iranian juvenile SLE patients. Furthermore, as it was not associate with adult SLE in Iranian population $^{9}$, rs7601754 SNP in STAT4 gene also had no significant association with Iranian juvenile SLE patients.

Complete deficiency of Complement C4 component has been shown to be strong genetic risk factor for SLE development ${ }^{28}$. In a recently performed study, low copy number variations (CNVs) of $\mathrm{C} 4, \mathrm{C} 4 \mathrm{~A}$ and $\mathrm{C} 4 \mathrm{~B}$ genes were identified to be associated with a stronger risk for JSLE, but not for adult SLE 29. Additionally, different classifications of SLE patients (juvenile-, adult-, and late- onset SLE) demonstrate different clinical and serological manifestations. JSLE patients show more severe disease activity and more frequent renal involvement in comparison to other SLE types 30. Considering discriminated manifestations of JSLE with regard to other SLE categories, genetic background plays a major role in the early development of autoimmune diseases, it is postulated that different genes possibly play distinct roles in adult- or juvenile-onset SLE. Cases of JSLE seem to be more severe than adult-onset disease, possibly because of genetic influences, but it is not more-detailed information about the molecular heterogeneity of the disease.

To sum up all the facts, this was the first attempt to evaluate the association of STAT4 gene SNPs with juvenile-SLE in Iranian population. While the rs7574865 SNP of STAT4 gene has been associated with adult SLE in both Iranian and other populations, it was not regarded as genetic contributing factor toward JSLE in our patient group. Moreover, rs7601754 SNP of this gene did not have relation with JSLE, as it was not for adult SLE through previous surveys. Therefore, the risk of JSLE was not under the impression of genetic variants in STAT4 gene. However, with regard to small number of the patients in the case group of this study, further investigations with satisfactory sample sizes and in other populations, as well as assessing other potential polymorphisms will be helpful for interpreting our observations to come up with a comprehensive conclusion of STAT4 role in JSLE etiopathogenesis. Therefore, the lack of association could be due to small number of patients in the study group.

\section{Acknowledgement}

This study was funded by Deputy of Research, Tehran University of Medical Sciences (grant number 94-03-41-30267).

\section{REFERENCES}

1. FENG PH. Systemic Lupus Erythematosus. Ann N Y Acad Sci 2007; 1108: 114-120.

2. Narchi H, Amirlak I. Juvenile Systemic Lupus Erythematosus. Curr Pediatr Rev 2007; 3: 225-232.

3. Murphy G, Isenberg D. Effect of gender on clinical presentation in systemic lupus erythematosus. Rheumatol 2013; 52: 2108-2115.

4. Kaplan MH. STAT4. Immunol Res 2005; 31: 231-241.

5. Mathur AN, Chang H-C, Zisoulis DG, Stritesky GL, Yu Q, O'Malley JT, et al. Stat3 and Stat4 direct 
development of IL-17-secreting Th cells. J Immunol 2007; 178: 4901-4907.

6. Hinks A, Cobb J, Marion MC, et al. Dense genotyping of immune-related disease regions identifies 14 new susceptibility loci for juvenile idiopathic arthritis. Nature Genetics 2013; 45: 664-669.

7. Liang YL, Wu H, Shen X, et al. Association of STAT4 rs7574865 polymorphism with autoimmune diseases: a meta-analysis. Mol Biol Rep 2012; 39: 8873-8882.

8. Yuan H, Feng JB, Pan HF, et al. A meta-analysis of the association of STAT4 polymorphism with systemic lupus erythematosus. Mod Rheumatol 2010; 20: 257262.

9. Mirkazemi S, Akbarian M, Jamshidi AR, et al. Association of STAT4 rs7574865 with susceptibility to systemic lupus erythematosus in Iranian population. Inflammation 2013; 36: 1548-1552.

10. Tong G, Zhang X, Tong W, Liu Y. Association between polymorphism in STAT4 gene and risk of rheumatoid arthritis: a meta-analysis. Hum Immunol 2013; 74 : 586-592.

11. Zamani MR, Salmaninejad A, Akbari Asbagh F, Masoud A, Rezaei N. STAT4 single nucleotide gene polymorphisms and susceptibility to endometriosisrelated infertility. Eur J Obstet Gynecol Reprod Biol 2016; 203: 20-24.

12. Hochberg MC. Updating the American College of Rheumatology revised criteria for the classification of systemic lupus erythematosus. Arthritis \& Rheum 1997; 40: 1725-1725.

13. Shi YY, He L. SHEsis, a powerful software platform for analyses of linkage disequilibrium, haplotype construction, and genetic association at polymorphism loci. Cell Res 2005; 15: 97-98.

14. Watford WT, Hissong BD, Bream JH, Kanno Y, Muul L, O'Shea JJ. Signaling by IL-12 and IL-23 and the immunoregulatory roles of STAT4. Immunol Rev 2004; 202: $139-156$.

15. Iwakura $\mathrm{Y}$, Ishigame $\mathrm{H}$. The IL-23/IL-17 axis in inflammation. J Clin Invest 2006; 116: 1218-1222.

16. Ghodke-Puranik Y, Niewold TB. Immunogenetics of systemic lupus erythematosus: A comprehensive review. J Autoimmun 2015; 64: 125-136.

17. Ziaee V, Tahghighi F, Moradinejad $\mathrm{MH}$, et al. Interleukin-6, interleukin-1 gene cluster and interleukin-1 receptor polymorphisms in Iranian patients with juvenile systemic lupus erythematosus. Eur Cytokine Netw 2014; 25: 35-40.

18. Pontillo A, Reis EC, Liphaus BL, Silva CA, CarneiroSampaio M. Inflammasome polymorphisms in juvenile systemic lupus erythematosus. Autoimmunity 2015; 48: 434-437.

19. Rezaei A, Ziaee V, Sharabian FT, et al. Lack of association between interleukin-10, transforming growth factor-beta gene polymorphisms and juvenileonset systemic lupus erythematosus. Clin Rheumatol 2015; 34: 1059-1064.
20. Zamani MR, Asbagh FA, Massoud AH, Salmaninejad A, Massoud A, Rezaei N. Association between a PD-1 gene polymorphism and antisperm antibody-related infertility in Iranian men. J Assist Reprod Genet 2015; 32: 103-106.

21. Mahmoudi M, Rezaiemanesh A, Salmaninejad A, et al. PDCD1 single nucleotide genes polymorphisms confer susceptibility to juvenile-onset systemic lupus erythematosus. Autoimmunity 2015; 48: 488-493.

22. Bahrami T, Soltani S, Moazzami K, et al. Association of PTPN22 gene polymorphisms with susceptibility to juvenile idiopathic arthritis in Iranian population. Fetal Pediatr Pathol 2017; 36: 42-48.

23. Baca V, Velazquez-Cruz R, Salas-Martinez G, EspinosaRosales F, Saldana-Alvarez Y, Orozco L. Association analysis of the PTPN22 gene in childhood-onset systemic lupus erythematosus in Mexican population. Genes Immun 2006; 7: 693-695.

24. Mahmoudi M, Tahghighi F, Ziaee V, et al. Interleukin-4 single nucleotide polymorphisms in juvenile systemic lupus erythematosus. Int J Immunogenet 2014; 41: 512-517.

25. Mosaad YM, Hammad A, Fawzy Z, et al. C1q rs292001 polymorphism and $\mathrm{Clq}$ antibodies in juvenile lupus and their relation to lupus nephritis. Clin Exp Immunol 2015; 182: 23-34.

26. Zamani MR, Aslani S, Salmaninejad A, Javan MR, Rezaei N. PD-1/PD-L and autoimmunity: A growing relationship. Cell Immunol 2016; 310: 27-41.

27. Ji JD, Lee WJ, Kong KA, et al. Association of STAT4 polymorphism with rheumatoid arthritis and systemic lupus erythematosus: a meta-analysis. Mol Biol Rep 2010; 37: 141-147.

28. Yang Y, Chung EK, Wu YL, et al. Gene copy-number variation and associated polymorphisms of complement component $\mathrm{C} 4$ in human systemic lupus erythematosus (SLE): low copy number is a risk factor for and high copy number is a protective factor against SLE susceptibility in European Americans. Am J Hum Genet 2007; 80: 1037-1054.

29. Pereira KM, Faria AG, Liphaus BL, et al. Low C4, $\mathrm{C} 4 \mathrm{~A}$ and $\mathrm{C} 4 \mathrm{~B}$ gene copy numbers are stronger risk factors for juvenile-onset than for adult-onset systemic lupus erythematosus. Rheumatology (Oxford) 2016; 55: 869-873.

30. Choi JH, Park DJ, Kang JH, et al. Comparison of clinical and serological differences among juvenile-, adult-, and late-onset systemic lupus erythematosus in Korean patients. Lupus 2015: 24: 1342-1349. 